\title{
Neonatal mortality in Ethiopia: a protocol for systematic review and meta-analysis
}

\author{
Tesfalidet Tekelab ${ }^{1,2^{*}}$, Mohammed Akibu ${ }^{3}$, Negash Tagesse ${ }^{4}$, Temesgen Tilhaun ${ }^{1}$, Yosef Yohanes ${ }^{5}$ \\ and Smriti Nepal ${ }^{2}$
}

\begin{abstract}
Background: A child's risk of dying is highest in the neonatal period, i.e. the first 28 days of life. Newborn death accounts for nearly half of under-five death. More than $80 \%$ of newborn deaths are the result of preventable and treatable conditions. Ethiopia has made significant progress towards reducing under-five mortality; however, the rate of neonatal mortality (NMR) still accounts for $41 \%$ of under-five deaths. With this systematic review and metaanalysis, we aim to determine the magnitude, causes, and determinants of neonatal mortality in Ethiopia.

Methods: We will conduct a comprehensive search of the following electronic databases: PubMed, MEDLINE, EMBASE, CINAHL, Google Scholar, and maternity and infant care databases as well as grey literature. We will assess the quality of studies by using Newcastle-Ottawa Scale (NOS) checklist. Two reviewers will screen all retrieved articles, conduct data extraction, and then critically appraise all identified studies. We will analyse data by using STATA 11 statistical software. We will demonstrate pooled estimates and determinants of neonatal mortality with effect size and 95\% confidence interval.

Discussion: The result from this systematic review will inform and guide health policy planners and researchers on the burden, causes, and determinants of neonatal mortality in Ethiopia. To our knowledge, this is the first systematic review in Ethiopia. We will synthesise the findings to generate up-to-date knowledge on neonatal mortality in Ethiopia.
\end{abstract}

\section{Systematic review registration: PROSPERO-CRD42018099663}

Keywords: Neonatal mortality, Causes of neonatal mortality, Meta-analysis, Ethiopia

\section{Background}

Child survival should remain at the heart of global health and development goals [1]. Globally, 2.6 million newborns die within the first month of life, annually. Newborn death accounts for nearly half of under-five deaths [2]. Even though there is a global decrease in neonatal mortality, the decrease is slower in neonatal mortality rates (NMRs) compared to under-five mortality rates $[1,2]$. Approximately 7000 newborns die daily; most of these deaths occur within the first week with one million taking their first and last breaths on the day they are born and one million dying within the next 6 days [3, 4]. Most newborn deaths (99\%) occur in

\footnotetext{
* Correspondence: ttesfalove@gmail.com

${ }^{1}$ Institute of Health sciences, Wollega University, Nekemte, Ethiopia

${ }^{2}$ School of Medicine and Public Health, University of Newcastle, Newcastle, Australia

Full list of author information is available at the end of the article
}

low-income countries with half occurring at home [5]. Sub-Saharan Africa and South Asia each account for $39 \%$ of all global neonatal deaths [3, 6]. In sub-Saharan Africa, the NMR is 31 per 1000 live births whereas the global NMR is 20 per 1000 live births [7]. Newborn deaths can be prevented through effective strategies such as skin-to-skin contact, early breast feeding initiation, newborn resuscitation, kangaroo mother care for premature babies, clean water, disinfectant, and good nutrition along with access to well-trained healthcare providers [8]. More than $80 \%$ of newborn deaths are the result of premature birth complication and infections such as sepsis, meningitis, and pneumonia $[3,4,9]$.

Ethiopia has made remarkable progress in achieving many of the national health indicators. The implementation of the National Child Survival Strategy (2005-2015) helped in the reduction of child mortality; however, the current under-five and neonatal mortality rates remain 
unacceptably high [10]. Between 2000 and 2016, under-five mortality in Ethiopia decreased from 166 to 67 per 1000 live births (reduction of 60\%). However, NMR is decreasing at a slower rate and now accounts for $41 \%$ of under-five deaths [11-13]. The leading causes for neonatal death in Ethiopia are prematurity, asphyxia, and neonatal sepsis $[10,14,15]$.

Researchers have stated that neonatal mortality rate to be a standard indicator for evaluation of health status of a country $[16,17]$. Therefore, it is important to explore the factors that contribute to neonatal death. Moreover, identifying the cause of death and cause-specific contributions to neonatal mortality is important in selecting strategies to further reduce newborn deaths. Studies have shown that neonatal mortality is influenced by several factors $[15,18]$. None of the studies have shown pooled estimates of neonatal mortality and its determinants in Ethiopia. Thus, there is a strong need to systematically evaluate the existing evidence on determinants of neonatal mortality on which one can base health policy planning.

\section{Objectives and research questions}

The objectives of this study are (1) to show the pooled prevalence of neonatal mortality in Ethiopia and (2) to synthesise evidence on the determinants and causes of neonatal mortality in Ethiopia.

This systematic review and meta-analysis is guided by the following research questions:

(1) What is the pooled estimate of neonatal mortality in Ethiopia? (2) What are the determinants and causes of neonatal mortality in Ethiopia?

\section{Methods}

\section{Reporting of the review findings}

We developed this protocol in accordance with the Preferred Reporting Items for Systematic review and Meta-analysis Protocols (PRISMA-P) statement [19] (Additional file 1). The protocol for this review was registered on PROSPERO ("CRD42018099663") on 19 September 2018. We will use the Preferred Reporting Items for Systematic review and Meta-analyses (PRISMA-2009) statement to report the findings [20].

\section{Inclusion criteria}

We will include cross-sectional, case-control, and cohort studies that have reported on neonatal mortality and have been published in peer-reviewed journals. In addition, we will include findings from regional or national survey reports. We will include studies that have been conducted in Ethiopia. Our focus will be on studies reporting factors, causes, and the rates of neonatal mortality.

\section{Exclusion criteria}

We will exclude studies published in languages other than English. We will exclude studies that include stillbirth on neonatal mortality. Additionally, we will exclude case reports and expert opinions.

\section{PECO search guide}

Population: Live born neonates

Exposure: Predictors/determinants of neonatal mortality. The determinants are characteristics or exposures that increase the likelihood of neonatal mortality. These may be related to residence, maternal age, educational status, and antenatal exposure.

Comparison: The reported reference group for each predictor/determinant in each study (e.g. neonatal mortality in mother living in urban area versus mothers living in rural area).

Outcome: Neonatal mortality is defined as 'a death occurring during the first four weeks ( 28 days) after birth of live baby' [21]. We will include studies that assess perinatal mortality, if the studies have excluded stillbirth from perinatal mortality.

\section{Search strategy}

We will develop an appropriate and comprehensive search strategy with relevant search terms and pilot test it before the final search. We will search PubMed, MEDLINE, EMBASE, CINAHL, Google Scholar, and Ovid Maternity and Infant Care Databases. We will include articles published from start of indexing until 10 August 2018. We will use Medical Subject Heading (Mesh), keywords, and free text search terms. As the search terms, we will include alternative terms for neonatal mortality, and will combine them using Boolean operators. To ensure the comprehensiveness, we will consult an expert librarian. The search strategy for PubMed is supplemented with this protocol (Additional file 2).

We will utilise snowballing to screen the references of identified articles for potentially relevant studies. Furthermore, we will contact experts, researchers, and relevant organisations for suggestions on other existing relevant studies.

\section{Selection of studies}

Two authors (TT and NT) will review the studies, based on inclusion and exclusion criteria. The review will follow three stages. During the first stage, we will assess the titles of the studies identified from the search. Then abstract screening, abstracts of these selected titles will be included for the final stage of full-text screening. During full-text screening, we will screen the full texts of abstracts selected in the previous stage. In the review, we will only include those studies approved by both authors. The authors will resolve disagreements through 
discussion or consultation with a third reviewer (SN). We will provide reason for exclusion for all excluded studies. We will prepare a final list of articles for data extraction.

\section{Data extraction and management}

Using the Joanna Briggs Institute (JBI) data extraction form for experimental/observational studies [22], we will extract relevant data. We will pretest the data extraction form on four studies of each type, to ensure that it adequately facilitates the collection of all necessary data required for an effective systematic review and meta-analysis.

Two review authors (TT and NT) will extract the data independently. Discrepancies between data extractors will be discussed to reach consensus. If a consensus cannot be reached, the authors will consult a third reviewer $(\mathrm{SN})$. For each included articles, we will record the first author's last name, year of publication, the setting where the study was conducted, study design, study period, sample size, the response rate, the population, outcome definition, comparison groups, and the effect estimate.

\section{Quality assessment}

Three authors (TT, NT, and YY) will independently conduct quality assessment of included studies, by using the checklist of the Newcastle-Ottawa Scale (NOS) (Additional file 3) for cohort and case-control studies [23]. We will use the adapted version of NOS for cross-sectional studies. Based on NOS, we will award studies a maximum of four stars within the selection, two stars within comparability, and three stars within outcome categories.

\section{Data synthesis and analysis}

We will perform a narrative description of the study population, the studies included, the risk factors identified, and the cause for mortality as well as the outcome characteristics. We will use tables and figures to summarise the selected studies and results. Using STATA 11 statistical software, we will carry out the data entry and statistical analysis. We will demonstrate the pooled prevalence of neonatal mortality in Ethiopia. To examine the possible risk of publication bias, we will use funnel plots and Egger's test [24]. A $p$ value $<0.10$ will be considered indicative of statistically significant publication bias. If there is evidence of publication bias, we will use Duval and Tweedie's trim-and-fill method [25]. We will assess heterogeneity by using chi-squared test on Cochran's $Q$ statistic with a 5\% level of statistical significance [26] and $I^{2}$ statistic, assuming that $I^{2}$ value of $25 \%$, $50 \%$, and $75 \%$ being representative of low, moderate, and high heterogeneity, respectively [27]. We will use fixed effect model if the studies have similar methodology, same population, and study design. If not, then, we will utilise the random effect model. If the heterogeneity is significant $\left(I^{2}>50 \%\right)$, then we will conduct subgroup analyses and meta-regression to investigate sources of heterogeneity. If the meta-analysis is not possible, we will conduct narrative synthesis.

\section{Subgroup and sensitivity analyses}

Based on study design, sample size, regions or state, year of publication, quality of studies, and study settings, we will perform subgroup analysis. To conduct sensitivity analysis, we will assess the stability or robustness of the pooled estimates to outliers and the impact of individual studies.

\section{Discussion}

This review will provide a detailed summary of the evidence on factors and causes of neonatal mortality in Ethiopia. This review will be the first to synthesise available data on neonatal mortality. Findings of the review will fill an evidence gap in understanding the burden, risk factors, and causes of neonatal mortality in Ethiopia. The result from this review will inform health policy planners and researchers up-to-date data on neonatal mortality and provide direction in which factors the policy should focus to reduce neonatal mortality in Ethiopia.

\section{Dissemination plan}

The results of this systematic review and meta-analysis will be published in a peer-reviewed journal and presented at national and international research conferences.

\section{Additional files}

Additional file 1: PRISMA-P (Preferred Reporting Items for Systematic review and Meta-Analysis Protocols) 2015 checklist: recommended items to address in a systematic review protocol. (DOCX $23 \mathrm{~kb}$ )

Additional file 2: PubMed search string. (DOCX $12 \mathrm{~kb}$ )

Additional file 3: Table S1. Methodological quality assessment of cohort studies using Newcastle-Ottawa Scale (NOS). (DOCX 15 kb)

Abbreviation

JBI: Joanna Briggs Institute; NOS: Newcastle-Ottawa scale; PECO: Population, Exposure, Comparison, and Outcome

Acknowledgements

We would like to acknowledge librarian who helped on the development of search string.

Funding

No funding was used.

Availability of data and materials

There was no primary data collection.

Authors' contributions

$\Pi \pi$ conceived and designed the protocol. $\Pi T$ wrote the first draft. $\Pi T$ designed search strategy. TT, MA, NT, TTB, YY \& SN wrote and developed the protocol. TT \& SN revised the protocol. All authors read and approved the final protocol. 


\section{Authors' information}

$\Pi T$ is a PhD candidate at the University of Newcastle, Australia, and lecturer of Reproductive and Maternal Health , Institute of Health Sciences, Wollega University, Ethiopia.

MA is lecturer at Department of Midwifery, Institute of Medicine and Health sciences, Debre Berhan University, Ethiopia.

NT is a paediatrician and lecturer at Department of Paediatrics, Hawassa University, Ethiopia.

$\Pi$ is an associate professor of obstetrician and gynaecology at the Institute of Health Sciences, Wollega University, Ethiopia.

YY is lecturer at Hawassa College of Health Science, Hawassa, Ethiopia. $\mathrm{SN}$ is PhD candidate at School of Medicine and Public Health, University of Newcastle, Australia.

\section{Ethics approval and consent to participate}

Not applicable.

\section{Consent for publication}

Not applicable, since the protocol does not contain any individual person's data.

\section{Competing interests}

The authors declare that they have no competing interests.

\section{Publisher's Note}

Springer Nature remains neutral with regard to jurisdictional claims in published maps and institutional affiliations.

\section{Author details}

Institute of Health sciences, Wollega University, Nekemte, Ethiopia. ${ }^{2}$ School of Medicine and Public Health, University of Newcastle, Newcastle, Australia. ${ }^{3}$ Department of Midwifery, Institute of Medicine and Health Sciences, Debre Berhan University, Debre Berhan, Ethiopia. ${ }^{4}$ Department of Paediatrics, Hawassa University, Hawassa, Ethiopia. ${ }^{5}$ Hawassa College of Health Science, Hawassa, Ethiopia.

Received: 26 August 2018 Accepted: 1 April 2019

Published online: 26 April 2019

\section{References}

1. Bryce J, Victora CG, Black RE. The unfinished agenda in child survival. Lancet. 2013:382:1049-59.

2. WHO. Children: reducing mortality.Key facts. 2017.

3. UNICEF. Every child alive; rhe urgent need to end newborn deaths. Genève, Switzerland. 2018.

4. United Nations Inter-agency Group for Child Mortality Estimation (UN IGME) Levels and trends in child mortality: report 2017. New York: United Nations Children's Fund; 2017.

5. Lawn JE, Cousens S, Zupan J, Lancet Neonatal Survival Steering Team. 4 million neonatal deaths: when? Where? Why? Lancet. 2005:365(9462):891-900.

6. UNICEF, World Health Organization, World Bank, United Nations Population Division. Levels and trends in child mortality: estimates developed by the UN Inter-agency Group for child mortality estimation UN IGME. 2014.

7. UNICEF, World Health Organization, World Bank, United Nations Population Division. Levels and trends in child mortality - report 2013. In: Estimates developed by the UN inter-agency group for child mortality estimation. New York: UNICEF. p. 2013.

8. Shefali O, Joy EL, Daniel RH, Colin M, Simon NC. Neonatal cause of death estimates for the early and late neonatal periods for 194 countries: 2000 2013. Bull World Health Organ. 2015;93:19-28.

9. $\mathrm{WHO}$ and Maternal and Child Epidemiology Estimation Group (MCEE). Estimates for child causes of death 2000-2016. 2018.

10. Federal Ministry of Health. National strategy for newborn and child survival in Ethiopia, 2015/16 - 2029/20 Addis Ababa, Ethiopia June 2015.

11. Central Statistical Agency (CSA) [Ethiopia] and ICF International. Ethiopia demographic and health survey 2016: key indicators report: Addis Ababa, Ethiopia, and Rockville, Maryland, USA. CSA and ICF.; 2016.

12. Central Statistical Agency [Ethiopia] and ICF International. Ethiopia demographic and health survey (EDHS) 2011. ORC Macro, Calverton, Maryland, USA. 2012:93-99.

13. UNICEF. Levels and trends in child mortality. New York: UNICEF; 2015.
14. Liu L, Oza S, Hogan D, Chu Y, Perin J, Zhu J, et al. Global, regional, and national causes of under-5 mortality in 2000-15: an updated systematic analysis with implications for the sustainable development goals. Lancet. 2016;388(10063):3027-35.

15. Mengesha HG, Sahle BW. Cause of neonatal deaths in Northern Ethiopia: a prospective cohort study. BMC Public Health. 2017;17(1):62.

16. Gonzalez RM, Gilleskie D. Infant mortality rate as a measure of a country's health: a robust method to improve reliability and comparability. Demography. 2017:54(2):701-20.

17. Yu VY. Global, regional and national perinatal and neonatal mortality. J Perinat Med. 2003:31(5):376-9.

18. Mekonnen Y, Tensou B, Telake DS, Degefie T, Bekele A. Neonatal mortality in Ethiopia: trends and determinants. BMC Public Health. 2013;13:483.

19. Moher D, Shamseer L, Clarke M, Ghersi D, Liberati A, Petticrew M, et al. Preferred reporting items for systematic review and meta-analysis protocols (PRISMA-P) 2015 statement. Syst Rev. 2015;4(1).

20. Moher D, Liberati A, Tetzlaff J, Altman DG. Preferred reporting items for systematic reviews and meta-analyses: the PRISMA statement. BMJ. 2009; 339:b2535.

21. WHO. World Health Organisation: Neonatal and perinatal mortality: country, regional and global estimates. WHO 20 Avenue Appia, 1211 Geneva 27, Switzerland. 2006

22. Joanna Briggs Institute. Joanna Briggs Institute reviewers' manual. Australia: the University of Adelaide. 2014 edition.

23. Wells G, Shea B, O'Connell D, Peterson J, Welch V, Losos M, et al. The Newcastle-Ottawa Scale (NOS) for assessing the quality of nonrandomised studies in meta-analyses. 2000.

24. Egger M, Smith GD. Meta-analysis. Potentials and promise. BMJ (Clinical research ed). 1997;315(7119):1371-4.

25. Duval S, Tweedie R. Trim and fill: a simple funnel-plot-based method of testing and adjusting for publication bias in meta-analysis. Biometrics. 2000; 56(2):455-63.

26. Cochran GW. The combination of estimates from different experiments. Biometrics. 1954;10:101-29.

27. Higgins JP, Thompson SG. Quantifying heterogeneity in a meta-analysis. Stat Med. 2002;21(11):1539-58
Ready to submit your research? Choose BMC and benefit from:

- fast, convenient online submission

- thorough peer review by experienced researchers in your field

- rapid publication on acceptance

- support for research data, including large and complex data types

- gold Open Access which fosters wider collaboration and increased citations

- maximum visibility for your research: over $100 \mathrm{M}$ website views per year

At BMC, research is always in progress.

Learn more biomedcentral.com/submission 